\title{
The ocular hypotensive effect of saffron extract in primary open angle glaucoma: a pilot study
}

\author{
Mohammad Hossein Jabbarpoor Bonyadi ${ }^{1,2^{*}}$, Shahin Yazdani ${ }^{3}$ and Saeed Saadat ${ }^{4}$
}

\begin{abstract}
Background: The progressive nature of glaucoma and its growing incidence make its therapy an important target for research. The role of oxidative damage in the pathogenesis of glaucoma makes antioxidants such as saffron extract an attractive target for potential clinical use. Herein, we evaluate the effect of aqueous saffron extract on intraocular pressure (IOP) in eyes with primary open-angle glaucoma (POAG).

Methods: Thirty-four eyes of 34 clinically stable POAG patients receiving treatment with timolol and dorzolamide eye drops were enrolled in this prospective, comparative, randomized interventional pilot study. Eligible subjects were randomized to receive $30 \mathrm{mg} /$ day aqueous saffron extract orally (17 subjects, 17 eyes) or placebo (17 subjects, 17 eyes) for one month as an adjunct to timolol and dorzolamide. Following treatment, both study groups entered a one-month wash-out period. The main outcome measure was IOP during treatment and after the wash-out period.
\end{abstract}

Results: Mean baseline IOP was $12.9 \pm 3.7$ versus $14.0 \pm 2.5 \mathrm{mmHg}$ in the saffron and control groups, respectively $(p=0.31)$. After three weeks of treatment, IOP was significantly decreased to $10.9 \pm 3.3 \mathrm{mmHg}$ in the saffron group as compared to $13.5 \pm 2.3 \mathrm{mmHg}$ in the control group $(p=0.013)$. At four weeks, IOP was still significantly lower in the saffron group ( $10.6 \pm 3.0$ versus $13.8 \pm 2.2 \mathrm{mmHg}, \mathrm{p}=0.001$ ). At the end of the wash-out period, IOP was $12.9 \pm 3.0 \mathrm{in}$ the saffron group versus $14.2 \pm 2.0 \mathrm{mmHg}$ in the control group $(p=0.175)$. None of the patients experienced side effects during the study and wash-out period.

Conclusions: Oral aqueous saffron extract seems to exert an ocular hypotensive effect in primary open-angle glaucoma. This effect became evident after three weeks of therapy.

The current study was registered at the International Clinical Trials Registry Platform (ICTRP) as IRCT201201278832N1.

Keywords: Open angle glaucoma, Saffron extract, Hypotensive effect, Antioxidative effect

\section{Background}

Glaucoma is the most common progressive optic neuropathy [1] and the most important risk factor for development of glaucomatous optic neuropathy is elevated intraocular pressure (IOP) [2]. Abnormal resistance to the outflow of aqueous humor through the trabecular meshwork (TM) is the key pathogenic factor in primary open angle glaucoma (POAG) [3].

Oxidative stress has been suggested to play a role in POAG and the trabecular meshwork is the most sensitive anterior chamber structure to oxidative stress [4-8].

\footnotetext{
* Correspondence: mhbonyadi@yahoo.com

'Department of Ophthalmology, Gonabad University of Medical Sciences, Gonabad, Iran

${ }^{2}$ Center of Excellence for Biodiversity, Faculty of Natural Sciences, University of Tabriz, Tabriz, Iran

Full list of author information is available at the end of the article
}

The important role of oxidative damage in the pathogenesis of POAG makes it an important therapeutic target and antioxidative therapy has been shown to entail an ocular hypotensive effect against dexamethasone induced IOP elevation [9].

Saffron, derived from the pistils of Crocus sativus, contains high concentrations of crocin and crocetin, which are carotenoid derivatives. This plant belongs to the Iridaceae family. Multiple divalent carbon bonds in saffron compounds confer its powerful radical scavenging, antioxidative and anti-tumor properties [10-13]. Saffron has been widely used for many years in traditional Asian medicine and in Persian medicine in particular to treat depression [14].

The purpose of this pilot study was to investigate the possible ocular hypotensive effect of high dose oral 
aqueous saffron extract on IOP in POAG. To the best of our knowledge this is the first report on this subject.

\section{Methods}

\section{Setting}

This randomized study was performed at the department of Ophthalmology, 22nd of Bahman Hospital, Gonabad, Iran in collaboration with the Ophthalmic Research Center, Shahid Beheshti University of Medical Sciences, Tehran, Iran. Consecutive POAG patients were recruited from the ophthalmology clinic and assessed for eligibility. Patients who met the inclusion criteria entered our pre-study observation period (February 2012March 2013) during which stability of glaucoma was confirmed (for at least 6 months) while the subjects were receiving topical medications. Eventually 50 eyes of the same number of stable POAG patients were enrolled for intervention (April 2013). This study was registered at the International Clinical Trials Registry Platform (ICTRP) as IRCT201201278832N1.

\section{Inclusion and exclusion criteria}

Patients with POAG older than 50 years of age who were under treatment with topical timolol $0.5 \%$ twice daily and dorzolamide $2 \%$ three times daily and were judged to have stable glaucoma control for at least 6 months based on a series of visual field and optic nerve head examinations were considered for enrollment as stated earlier.

Exclusion criteria consisted of prior intraocular surgery, glaucoma other than POAG, visual acuity less than $5 / 10$, advanced glaucoma (equal or more than 90 percent vertical cupping), history of saffron hypersensitivity, intestinal absorption abnormalities and smoking. In the case of bilateral involvement, only the right eye was included. None of the patients were taking medications (e.g., chloroquine) known to interfere with carotenoid absorption. Patients with central corneal thickness (CCT) values less than 500 or more than 540 microns were also excluded.

During the study period, enrolled subjects would be eliminated from the study if they failed to attend regular follow-up sessions, maintain a regular regimen of topical glaucoma medication or saffron/placebo, or consumed a systemic medication with a known effect on IOP.

\section{Informed consent and ethical issues}

The study adhered to the tenets of the Declaration of Helsinki and was approved by the Ethics Committee of Gonabad Medical University. Prior to enrollment, potential advantages and risks of therapy were discussed with the patients and written informed consent was obtained from all participants. Consent forms contained possible side effects of high dose oral saffron including anxiety, appetite changes, nausea, headache, dry mouth, constipation, allergic reactions and jaundice.

\section{Pre-intervention evaluations and follow-up}

All participants underwent a detailed baseline ophthalmic examination including slitlamp biomicroscopy, gonioscopy, dilated lens and optic nerve head examination and IOP measurement using Goldmann applanation tonometry in addition to corneal pachymetry (OcuScan, Alcon Laboratories, Irvine, California, USA). For all cases and control patients, IOP was measured between 9 to $10 \mathrm{AM}$ at baseline, at 1, 3 and 4 weeks following initiation of intervention, and at the end of the wash-out period.The list of side effects as mentioned in Informed Consent and Ethical Issues section was checked in each session. Every patient was asked and examined according to mentioned list and also asked about any other symptom in each exam session to reveal possible side effects.

\section{Randomization and masking}

Patients were randomly assigned to the saffron regimen or placebo (simple randomization in two parallel groups). Both capsules were identical regarding shape, color and size. Participants were kept masked to the intervention and outcome measures, the ophthalmologist evaluating the outcome measure and the statistician involved in data analysis were also unaware of patient identity and study group. Eligible patients were introduced to our secretary who randomly selected one sealed, opaque envelope with 28 capsules containing saffron extract or placebo for each patient according to the randomization sheet.

The allocated group of each patient was registered and concealed up to the end of the study period and revealed only after final data analysis.

\section{Intervention}

Crocus sativus L. stigmas were collected from Gonabad (East Sorkhfam Saffron Co. Gonabad, Khorasan, Iran). Using the maceration method, saffron capsules containing thirty milligrams of the concentrated powder were prepared [12]. Starch powder was used with the same weight inside identical capsules for the placebo group. Capsule preparation for both study groups was performed by Genaati pharmacy, Gonabad, Khorasan, Iran. Study subjects were randomly assigned to oral saffron extract ( $30 \mathrm{mg} /$ day) versus placebo for a one month period while topical timolol and dorzolamide were continued throughout the randomization process. Following treatment, both groups entered a one-month wash-out period, during which all patients stopped ingestion of saffron/placebo capsules but continued their glaucoma medications as before. At the end of the wash-out period IOP measurement (using Goldmann applanation tonometry) and ophthalmologic evaluation (by a masked ophthalmologist MHJB) was 
repeated. During the study period patients were asked about and examined for possible side effects of saffron.

\section{Outcome measures}

The main outcome measure of the study was IOP.

\section{Statistical methods}

In this pilot RCT we enrolled 25 POAG patients in each group. Means and standard deviations were used for description of study parameters. Statistical analysis was performed by a biostatistician using SPSS software version 17.0 (SPSS Co., Chicago, IL, USA) who was unaware of study group identity. Mean CCT and mean IOP differences between study and control groups were analyzed individually by independent student t-test. The Mann-whitney test was employed to compare mean vertical cup disc ratio between the two groups because of nonnormal distribution of this variable.

\section{Results}

Initially 50 subjects fulfilled the eligibility criteria and were enrolled but a total of 16 subjects were eliminated from the study. The excluded patients in the saffron group consisted of 5 patients with incomplete follow-up and 3 patients who used their capsules irregularly. Excluded patients in the placebo group consisted of 6 patients with incomplete follow-up, one patient with irregular capsule consumption and another subject who started a systemic B blocker during the study period.

Eventually a total of 34 randomized subjects including 17 patients ( 7 female and 10 male individuals) in the saffron group and 17 other patients (6 female and 11male individuals) in the placebo group completed all followup sessions. None of the enrolled patients experienced side effects of saffron during the study or the wash-out period.

Mean patient age was $66.3 \pm 9.5$ (range 51 to 79 ) years versus $67.6 \pm 8.3$ (range 53 to 77 ) years in the saffron and placebo groups, respectively $(\mathrm{p}=0.69)$. CCT was $518 \pm 13.1$ microns in the saffron group versus $519 \pm 14.7$ microns in the control group $(\mathrm{p}=0.98)$. Mean vertical cup disc ratio was $0.68 \pm 0.10$ in the saffron group and $0.71 \pm 0.08$ in the placebo group $(\mathrm{P}=0.33)$. Mean baseline IOP was $12.9 \pm 3.7$ (range 7 to 19 ) $\mathrm{mmHg}$ in the saffron group and $14.0 \pm 2.5$ (range 9 to 18 ) $\mathrm{mmHg}$ in the control group (95\% confidence interval $[\mathrm{CI}]$ for mean difference:$1.1 \pm 2.2 \mathrm{mmHg}, \mathrm{p}=0.31$ ). Baseline features of enrolled patients are summarized in Table 1.

One week after intervention, IOP was $12.0 \pm 3.3$ (range 8 to 17 ) $\mathrm{mmHg}$ in the saffron group versus $13.6 \pm 2.6$ (range 9 to 18$) \mathrm{mmHg}$ in the control group (95\% CI for mean difference: $-1.65 \pm 2.05 \mathrm{mmHg}, \mathrm{p}=0.114$ ). At three weeks, IOP reached $10.9 \pm 3.3$ (range 6 to 17) $\mathrm{mmHg}$ in the saffron group versus $13.5 \pm 2.3$ (range 9 to 17 ) $\mathrm{mmHg}$ in
Table 1 Baseline characteristics of patients

\begin{tabular}{cccc}
\hline & Saffron group & \multicolumn{2}{c}{ Placebo group } \\
\hline Male & 10 & 11 & $\mathrm{p}^{\beta}=0.72$ \\
Female & 7 & 6 & \\
Age & $66.3 \pm 9.5$ & $67.6 \pm 8.3$ & $\mathrm{p}^{*}=0.69$ \\
CCT & $518 \pm 13.1$ & $519 \pm 14.7$ & $\mathrm{p}^{*}=0.98$ \\
vertical cup disc ratio & $0.68 \pm 0.10$ & $0.71 \pm 0.08$ & $\mathrm{P}^{\oplus}=0.33$ \\
VF MD & $-5.47 \pm 1.5$ & $-4.99 \pm 1.9$ & $\mathrm{p}^{*}=0.416$ \\
\hline
\end{tabular}

CCT: central corneal thickness, VF MD: visual field defect mean deviation.

$\beta$ : based on Chi-square test.

*: based on t-test.

๑: based on Mann-whitney test.

the control group (95\% CI for mean difference; $-2.6 \pm$ $2.00 \mathrm{mmHg}, \mathrm{p}=0.013)$. At four weeks IOP was $10.6 \pm 3.0$ (range 6 to 16) $\mathrm{mmHg}$ in the saffron group versus $13.8 \pm$ 2.2 (range 10 to17) $\mathrm{mmHg}$ in the control group (95\% CI for mean difference: $-3.25 \pm 1.85 \mathrm{mmHg}, \mathrm{p}=0.001)$. At the end of the wash-out period, IOP was $12.9 \pm 3.0$ (range 8 to 18 ) $\mathrm{mmHg}$ in the saffron group and $14.2 \pm 2.0$ (range 11 to 18 ) $\mathrm{mmHg}$ in the control group (95\% CI for mean difference: $-1.20 \pm 1.80 \mathrm{mmHg}, \mathrm{p}=0.175$ ) (Figure 1).

\section{Discussion}

Saffron has been widely used for many years in traditional Persian medicine to treat depression [14] and its crude extract has recently been shown to prevent tumor formation [13] and selenite-induced cataracts [15].

Saffron belongs to the Iridaceae family and its variants are not of the same quality and strength. Strength is related to several factors including the amount of styles picked along with the red stigma. Grades of Iranian saffron are: "Sargol" (red stigma tips only, premium grade), "Pushal" or "Pushali" (red stigmas plus some yellow style, lower strength), "Bunch" saffron (red stigmas plus large amounts of yellow style, presented in a tiny bundle) and "Konge" (yellow style only, claimed to have aroma but with very little, if any, coloring potential). In our study only premium Sargol grade fresh saffron was used by East Sorkhfam Saffron Co. for capsule preparation.

Antioxidants, ingested through diet and supplements, have been shown to be beneficial in terms of reducing the risk of multiple ocular diseases including age-related macular degeneration (based on the Age-Related Eye Disease Study, AREDS) [16] and cataracts [17].

A number of studies have strongly suggested the role of oxidative stress in POAG [4-8,18,19] and there is increasing evidence that oxidative stress also has an important role in progression of glaucomatous damage [20]. The TM is the most sensitive anterior chamber tissue to oxidative stress. [4] Light induced formation of oxidative radicals may target the TM and contribute to the pathogenesis of glaucoma [4,21]. Disturbed TM 


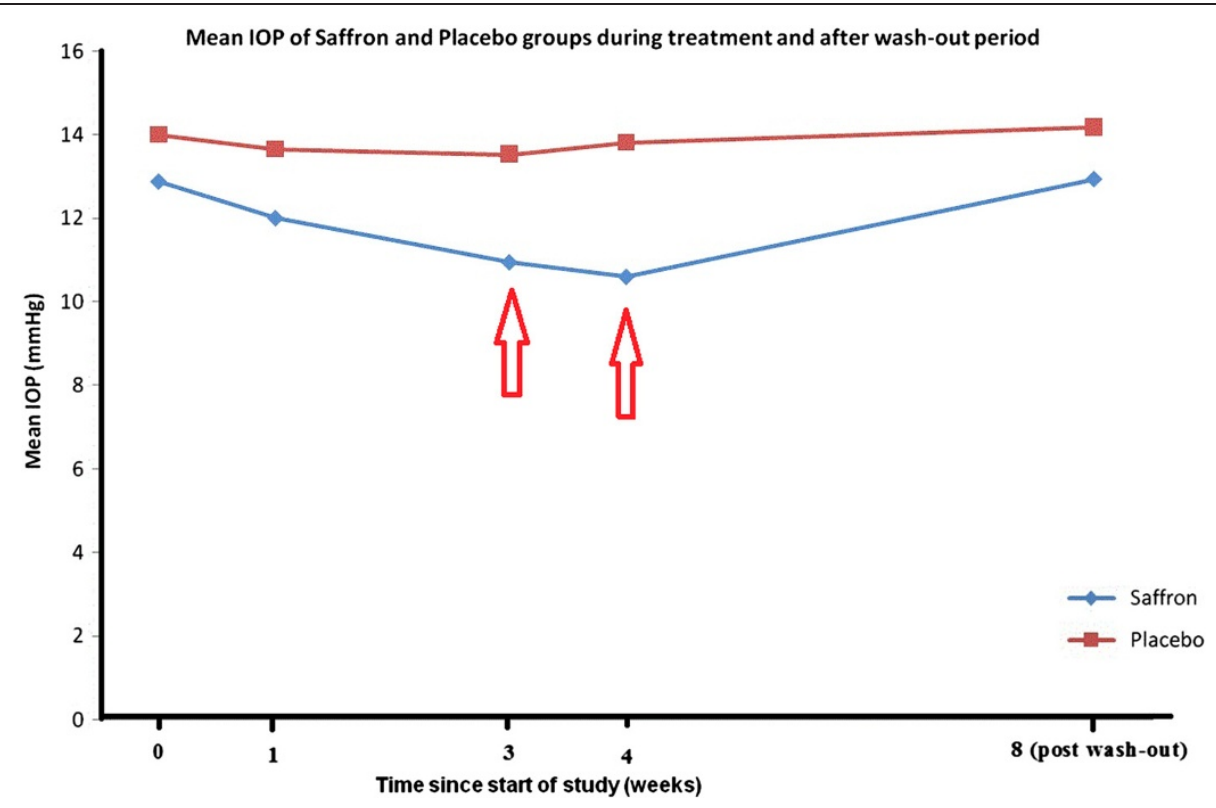

Figure 1 Mean IOP change during 4 weeks after initiation of saffron or placebo in addition to conventional timolol and dorzolamide therapy in primary open angle glaucoma and after one month of wash-out (Arrows indicate where mean IOP levels were significantly different between the two study groups).

cell homeostasis by oxidative stress may lead to cellular loss and structural alterations in its extracellular matrix resulting in impaired aqueous humor outflow and thereby an increase in IOP [22,23]. In addition to the observed effect of saffron extract regarding IOP reduction, its possible role as antioxidant-neuroprotective agent [24] may further provide protection against glaucomatous optic neuropathy.

Other agents with antioxidant properties have been used for treatment of glaucoma. Ginkgo biloba, similar to saffron, has antiapoptotic and antioxidant capacities $[25,26]$ and has demonstrated desirable effects on steroidinduced ocular hypertension. In a rabbit model, Jia et al [9] demonstrated that topical Ginkgo biloba extract 4 . times daily could suppress dexamethasone induced IOP elevation after 14 days of treatment. In another in vitro study, use of vitamin $\mathrm{E}$ as an antioxidant agent was shown to reduce the production of reactive oxygen species and inhibit cell death in TM cell cultures derived from glaucomatous patients [27]. Oxidative stress, in vitro, has been shown to result in mitochondrial and cellular dysfunction in the TM resulting in trabecular cell apoptosis [27,28]. Therefore antioxidants seems to exert both a short term effect in terms of rehabilitating damaged but still functional TM, and a long term benefit by reducing apoptosis. The ocular hypotensive effect of saffron extract in our study could be due to the short term effect.

The current study showed that oral aqueous saffron extract had an ocular hypotensive effect in POAG patients receiving treatment with timolol and dorzolamide.
This effect became evident three weeks after therapy and was accentuated at four weeks.

Employing a washout period, IOP returned to pretreatment levels after one month of discontinuation. (Figure 1) The observed reduction in IOP may be due to antioxidant carotenoid derivatives present in saffron extract, mainly crocin and crocetin.

In a recently published epidemiologic study in an African-American population it has been shown that carotenoids seem to display a protective effect against glaucoma [29]. In contrast, Kang et al reported no association between higher intake of various antioxidants and glaucoma [30]. Such differences in the correlation between glaucoma risk and carotenoids may be due to differences in study population, age, eating habits and food preparation methods. It has also been shown that genetic predisposition to oxidative stress may determine susceptibility to glaucoma [18] and the response to antioxidants as ocular hypotensive agents seems to be influenced by genetic background.

In the current study, we selected POAG patients whose IOP was controlled with two topical medications. We assume that regarding the role of oxidative damage in the pathogenesis of glaucoma, our results may be generalizable for POAG of different severity levels and possibly other types of glaucoma. Although our observations are promising, there are limitations which have to be kept in mind; regular high dose crude oral saffron may not be a feasible treatment option for glaucoma while small sample size and short term follow-up in this 
pilot study are restrictions of our study. Future studies with larger sample size and longer follow-up on different types glaucoma with varying severity could verify our results.

Saffron shown to have a very low toxicity in in vivo studies and daily doses of up to $1.5 \mathrm{gr}$ are thought to be safe. Toxic effects are reported with 5 gr and above, with a lethal dose of approximately $20 \mathrm{gr}$ and it could induce abortion in doses $>10$ gr. The colored constituents may accumulate in sclera, skin and may thus mimic icteric complaints. Nausea was described in doses between 1.2 gr and $2 \mathrm{gr}$ followed by vomiting, diarrhea and bleeding [31]. Since the dose shown to be efficacious in depression trials corresponded to approximately $30 \mathrm{mg}$ of saffron, there is a large safety margin [32]".

\section{Conclusions}

In summary, this pilot study revealed an ocular hypotensive effect from high dose oral aqueous saffron extract in POAG patients when the supplement was added to conventional timolol and dorzolamide treatment. To the best of our knowledge this is the first study reporting the effect of saffron on IOP. This early ocular hypotensive effect, similar to its influence in early age-related macular degeneration, [33] indicates that high dose systemic saffron may entail a therapeutic effect.

It must be kept in mind that high dose antioxidants may have paradoxical pro-oxidative effects exemplified by an increased risk of lung cancer with higher dietary intake of carotene [34,35]. Whether topically administered saffron compounds may also reduce IOP, requires further studies. Reaching a topical saffron formulation necessitates preparing a topical solution with reduced coloring capacity and minimal sensitizing side effects.

\section{Abbreviations}

POAG: Primary open angle glaucoma; IOP: Intraocular pressure.

\section{Competing interests}

The authors declare that they have no competing interests.

\section{Authors' contributions \\ MHJB: Study design, Patient enrollment, Examination and data collection, Data analysis, Manuscript preparation. SY: Supervision on data acquisition, Interpretation and review of data, Final edition and approval of the manuscript. SS: Data analysis. Dr JB has full access to all of the data in the study and takes responsibility for the integrity of the data and the accuracy of the data analysis. None of authors has potential conflicts of interest, including relevant financial interests, activities or relationships. All authors read and approved the final manuscript.}

\section{Acknowledgement}

The current study was funded by Gonabad University of Medical Sciences. The authors would like to thank Mrs. Zahra Ramezani for registering and randomizing the patients and entering data.

\section{Author details}

${ }^{1}$ Department of Ophthalmology, Gonabad University of Medical Sciences, Gonabad, Iran. ${ }^{2}$ Center of Excellence for Biodiversity, Faculty of Natural Sciences, University of Tabriz, Tabriz, Iran. ${ }^{3}$ Ophthalmic Research Center, Shahid Beheshti University of Medical Sciences, Tehran, Iran. ${ }^{4}$ School of Medicine, Shahroud University of Medical Sciences, Shahroud, Iran.
Received: 2 October 2014 Accepted: 8 October 2014

Published: 15 October 2014

\section{References}

1. Quigley HA: Number of people with glaucoma worldwide. $\mathrm{Br} J$ Ophthalmol 1996, 80(5):389-393.

2. Sommer A: Intraocular pressure and glaucoma. Am J Ophthalmol 1989, 107(2):186-188.

3. Quigley HA: Open-angle glaucoma. N Engl J Med 1993, 328(15):1097-1106.

4. Izzotti A, Saccà SC, Longobardi M, Cartiglia C: Sensitivity of ocular anterior chamber tissues to oxidative damage and its relevance to the pathogenesis of glaucoma. Invest Ophthalmol Vis Sci 2009, 50(11):5251-5258.

5. Majsterek I, Malinowska K, Stanczyk M, Kowalski M, Blaszczyk J, Kurowska AK, Kaminska A, Szaflik J, Szaflik JP: Evaluation of oxidative stress markers in pathogenesis of primary open-angle glaucoma. Exp Mol Pathol 2011, 90(2):231-237.

6. Erdurmuş M, Yağcı R, Atış Ö, Karadağ R, Akbaş A, Hepşen IF: Antioxidant status and oxidative stress in primary open angle glaucoma and pseudoexfoliative glaucoma. Curr Eye Res 2011, 36(8):713-718.

7. Izzotti A, Bagnis A, Saccà SC: The role of oxidative stress in glaucoma. Mutat Res 2006, 612(2):105-114.

8. Ferreira SM, Lerner SF, Brunzini R, Evelson PA, Llesuy SF: Oxidative stress markers in aqueous humor of glaucoma patients. Am J Ophthalmol 2004, 137(1):62-69.

9. Jia LY, Sun L, Fan DS, Lam DS, Pang CP, Yam GH: Effect of topical Ginkgo biloba extract on steroid-induced changes in the trabecular meshwork and intraocular pressure. Arch Ophthalmol 2008, 126(12):1700-1706.

10. Assimopoulou AN, Sinakos Z, Papageorgiou VP: Radical scavenging activity of Crocus sativus L. extract and its bioactive constituents. Phytother Res 2005, 19(11):997-1000.

11. Ochiai T, Shimeno H, Mishima K, Iwasaki K, Fujiwara M, Tanaka H, Shoyama Y, Toda A, Eyanagi R, Soeda S: Protective effects of carotenoids from saffron on neuronal injury in vitro and in vivo. Biochim Biophys Acta 2007, 1770(4):578-584.

12. Hosseinzadeh H, Sadeghnia HR, Ziaee T, Danaee A: Protective effect of aqueous saffron extract (Crocus sativus $\mathrm{L}$.) and crocin, its active constituent, on renal ischemia reperfusion- induced oxidative damage in rats. J Pharm Pharm Sci 2005, 8(3):387-393.

13. Bolhassani A, Khavari A, Bathaie SZ: Saffron and natural carotenoids: Biochemical activities and anti-tumor effects. Biochim Biophys Acta 2014, 1845(1):20-30

14. Shahmansouri N, Farokhnia M, Abbasi SH, Kassaian SE, Noorbala Tafti AA, Gougol A, Yekehtaz H, Forghani S, Mahmoodian M, Saroukhani S, ArjmandiBeglar A, Akhondzadeh S: A randomized, double-blind, clinical trial comparing the efficacy and safety of Crocus sativus L. with fluoxetine for improving mild to moderate depression in post percutaneous coronary intervention patients. J Affect Disord 2014, 155:216-222.

15. Makri OE, Ferlemi AV, Lamari FN, Georgakopoulos CD: Saffron administration prevents selenite-induced cataractogenesis. Mol Vis 2013, 19:1188-1197.

16. Age-Related Eye Disease Study Group: A randomized, placebo- controlled, clinical trial of high-dose supplements with vitamins $\mathrm{C}$ and $\mathrm{E}$, betacarotene, and zinc for age related macular degeneration and vision loss: AREDS report no. 8. Arch Ophthalmol 2001, 119(10):1417-1436.

17. Ravindran RD, Vashist P, Gupta SK, Young IS, Maraini G, Camparini M, Jayanthi R, John N, Fitzpatrick KE, Chakravarthy U, Ravilla TD, Fletcher AE: Inverse association of vitamin $\mathrm{C}$ with cataract in older people in India. Ophthalmology 2011, 118(10):1958-1965.

18. Izzotti A, Saccà SC, Longobardi M, Cartiglia C: Mitochondrial damage in the trabecular meshwork of patients with glaucoma. Arch Ophthalmol 2010, 128(6):724-730.

19. Saccà SC, Izzotti A, Rossi P, Traverso C: Glaucomatous outflow pathway and oxidative stress. Exp Eye Res 2007, 84(3):389-399.

20. Tezel G, Wax MB: Glial modulation of retinal ganglion cell death in glaucoma. J Glaucoma 2003, 12(1):63-68.

21. Wood JP, Lascaratos G, Bron AJ, Osborne NN: The influence of visible light exposure on cultured RGC-5 cells. Mol Vis 2007, 14:334-344.

22. Zhou L, Li Y, Yue BY: Oxidative stress affects cytoskeletal structure and cell-matrix interactions in cells from an ocular tissue: the trabecular meshwork. J Cell Physiol 1999, 180(2):182-189.

23. Li AF, Tane N, Roy S: Fibronectin over expression inhibits trabecular meshwork cell monolayer permeability. Mol Vis 2004, 10:750-757. 
24. Maccarone R, Di Marco S, Bisti S: Saffron supplement maintains morphology and function after exposure to damaging light in mammalian retina. Invest Ophthalmol Vis Sci 2008, 49:1254-1261.

25. Eckert A, Keil U, Kressmann S, Schindowski K, Leutner S, Leutz S, Müller WE: Effects of EGb761 Gingko biloba extract on mitochondrial functions and oxidative stress. Pharmacopsychiatry 2003, 36(1):S15-S23.

26. Takuma K, Hoshina Y, Arai S, Himeno Y, Matsuo A, Funatsu Y, Kitahara Y, Ibi D, Hayase M, Kamei H, Mizoguchi H, Nagai T, Koike K, Inoue M, Yamada K: Ginkgo biloba extract EGb 761 attenuates hippocampal neuronal loss and cognitive dysfunction resulting from chronic restraint stress in ovariectomized rats. Neuroscience 2007, 149(2):256-262.

27. He Y, Leung KW, Zhang YH, Duan S, Zhong XF, Jiang RZ, Peng Z, TombranTink J, Ge J: Mitochondrial complex I defect induces ROS release and degeneration in trabecular meshwork cells of POAG patients: protection by antioxidants. Invest Ophthalmol Vis Sci 2008, 49(4):1447-1458.

28. Chen M, Liu B, Gao Q, Zhuo Y, Ge J: Mitochondria-targeted peptide MTP- 131alleviates mitochondrial dysfunction and oxidative damage in human trabecular meshwork cells. Invest Ophthalmol Vis Sci 2011, 1; 52(10):7027-7037.

29. Giaconi JA, Yu F, Stone KL, Pedula KL, Ensrud KE, Cauley JA, Hochberg MC, Coleman AL, Study of Osteoporotic Fractures Research Group: The association of consumption of fruits/vegetables with decreased risk of glaucoma among older African-American women in the study of osteoporotic fractures. Study of Osteoporotic Fractures Research Group. Am J Ophthalmol 2012. 154(4):635-644.

30. Kang JH, Pasquale LR, Willett W, Rosner B, Egan KM, Faberowski N, Hankinson SE: Antioxidant intake and primary open-angle glaucoma: a prospective study. Am J Epidemiol 2003, 158(4):337-346.

31. Schmidt M, Betti G, Hensel A: Saffron in phytotherapy: pharmacology and clinical uses. Wien Med Wochenschr 2007, 157(13-14):315-319.

32. Akhondzadeh S, Fallah-Pour H, Afkham K, Jamshidi AH, Khalighi-Cigaroudi F: Comparison of Crocus sativus L. and imipramine in the treatment of mild to moderate depression: a pilot double-blind randomized trial [ISRCTN45683816]. BMC Complement Altern Med 2004, 4:12.

33. Falsini B, Piccardi M, Minnella A, Savastano C, Capoluongo E, Fadda A, Balestrazzi E, Maccarone R, Bisti S: Influence of saffron supplementation on retinal flicker sensitivity in early age-related macular degeneration. Invest Ophthalmol Vis Sci 2010, 51(12):6118-6124.

34. Alpha-Tocopherol T, Group BCCPS: The effect of vitamin E and beta carotene on the incidence of lung cancer and other cancers in male smokers. N Engl J Med 1994, 330(15):1029-1035.

35. Omenn GS, Goodman GE, Thornquist MD, Balmes J, Cullen MR, Glass A, Keogh JP, Meyskens FL Jr, Valanis B, Williams JH Jr, Barnhart S, Cherniack MG, Brodkin CA, Hammar S: Risk factors for lung cancer and for intervention effects in CARET, the Beta-Carotene and Retinol Efficacy Trial. J Natl Cancer Inst 1996, 88(21):1550-1559.

doi:10.1186/1472-6882-14-399

Cite this article as: Jabbarpoor Bonyadi et al:: The ocular hypotensive effect of saffron extract in primary open angle glaucoma: a pilot study. BMC Complementary and Alternative Medicine 2014 14:399.

\section{Submit your next manuscript to BioMed Central and take full advantage of:}

- Convenient online submission

- Thorough peer review

- No space constraints or color figure charges

- Immediate publication on acceptance

- Inclusion in PubMed, CAS, Scopus and Google Scholar

- Research which is freely available for redistribution

Submit your manuscript at www.biomedcentral.com/submit
Ciomed Central 